\title{
Memory of Conditioned Taste Aversion Is Erased by Inhibition of PI3K in the Insular Cortex
}

\author{
Ilana Slouzkey', Kobi Rosenblum' and Mouna Maroun*,' \\ 'Sagol Department of Neurobiology, Faculty of Natural Sciences, University of Haifa, Haifa, Israel
}

\begin{abstract}
The conditioned taste aversion (CTA) paradigm, in which association between a novel taste and visceral malaise is formed, gives a unique experimental setting to examine the mechanisms underlying memory acquisition and extinction processes. AKT is a main kinase of the phosphoinositide 3-kinase cascade (PI3K) and has been implicated in long-term memory. We have recently reported that blockade of PI3K in the basolateral amygdala (BLA) before retrieval of fear memory was associated with long-term reduction in fear responses, suggesting a possible role of PI3K inhibition in fear erasure. In this study, we aimed to elucidate whether PI3K has a similar role in the insular cortex (IC), which has a crucial role in CTA acquisition, consolidation, maintenance, and extinction. To that end, we (I) monitored AKT phosphorylation in the IC following CTA acquisition and extinction and (2) inhibited PI3K by local microinjection of the PI3K inhibitor LY294002 at different stages of CTA acquisition and extinction. Our results show that while AKT phosphorylation is increased following CTA learning, it is decreased following CTA extinction. Inhibition of AKT phosphorylation in the IC before or after the first CTA retrieval test resulted in reduction in the aversion index. This reduction in aversion is due to the erasure of the original CTA trace memory, as re-application of the unconditioned stimulus (lithium chloride) did not induce the recovery of aversion in LY294002-treated animals. Our present data add new evidence to suggest that PI3K is engaged in consolidation of aversive memories, as its inhibition is associated with erasure of CTA memory.

Neuropsychopharmacology (2013) 38, II43-1 I53; doi:10.1038/npp.2013.20; published online 6 February 2013
\end{abstract}

Keywords: extinction; conditioned taste aversion; insular cortex; PI3K; erasure; consolidation

\section{INTRODUCTION}

Experimental extinction is the decline in the frequency or intensity of the conditioned response following the withdrawal of reinforcement (Berman and Dudai, 2001; Bouton and Nelson, 1994; Myers and Davis, 2002; Rescorla, 1996). Studies showed that the formation of long-term extinction memory is associated with activation of kinase cascades, transcription factors, and proteins (Berman et al, 2003; Berman and Dudai, 2001). The phosphoinositide 3-kinase (PI3K) has been found vital for cell proliferation and prevention of apoptosis. A well-documented PI3K downstream target is protein serine/threonine kinase AKT/PKB; the activation of AKT is usually dependent on PI3K, and its phosphorylation is blocked by PI3K inhibitors.

Increasing evidence indicates that PI3K has a role in mediating the formation of fear memory and its extinction (Lin et al, 2001, 2003; Chen et al, 2005; Kritman and Maroun, 2012).

We have recently shown that the effects of inhibition of PI3K in the basolateral amygdala (BLA) in contextual fear formation and its extinction are time dependent. Namely,

\footnotetext{
*Correspondence: Dr M Maroun, Sagol Department of Neurobiology, Faculty of Natural Sciences, University of Haifa, Haifa 31905, Israel, Tel: +972 48249657, E-mail: mmaroun@psy.haifa.ac.il or mouna.maroun@gmail.com

Received 26 July 20 12; revised 23 November 20 12; accepted 2 January 2013; accepted article preview online 16 January 2013
}

infusion of the PI3K inhibitor, LY294002, into the BLA following fear conditioning was associated with enhanced freezing and impaired extinction. In contrast, when injected before the retrieval of fear memory, it was associated with impaired recall and subsequent attenuation of fear memory (Kritman and Maroun, 2012). These findings join previously reported data to show that PI3K inhibition in the hippocampus or amygdala blocks fear memory formation and retrieval in rats (Barros et al, 2001; Chen et al, 2005; Opazo et al, 2003) and induces dephosphorylation of AKT in the amygdala following extinction (Lin et al, 2003). Together, these results point to a possible role of PI3K in inhibition of fear.

In this study, we aimed to generalize the role of PI3K in acquisition and extinction to another aversive memory not related to fear response. In conditioned taste aversion (CTA) rats learn to avoid a taste if the first encounter with it is followed by malaise (Bures et al, 1998; Bureš et al, 1988; Garcia et al, 1955, 1966; Revusky and Bedarf, 1967).

Similarly to fear-based memories, CTA memory is robust and long lasting (Bures et al, 1998; Fanselow and Gale, 2003; LeDoux et al, 1989; Nachman, 1963). Nevertheless, under certain conditions CTA memory can be extinguished (Berman and Dudai, 2001; Berman et al, 2003). The insular cortex (IC), which contains the gustatory cortex (Berman et al, 1998, 2000; Rosenblum et al, 1993), is involved in CTA acquisition and its extinction (Berman and Dudai, 2001; 
Eisenberg et al, 2003; Roman and Reilly, 2007; Roman et al, 2006). Hence, in the present study, we sought to examine the role of PI3K inhibition in the IC on CTA acquisition and extinction. Toward that end, we examined (1) changes in AKT phosphorylation following the different stages of CTA acquisition and extinction and (2) whether the PI3K inhibition in the IC disrupts the formation of aversive CTA memory and its extinction, and (3) whether the inhibition of $\mathrm{PI} 3 \mathrm{~K}$ has a role in the erasure of CTA memory.

\section{MATERIALS AND METHODS}

Male Sprague-Dawley rats ( $\sim 60$ days old, 250-300 g) were caged individually at $22 \pm 2{ }^{\circ} \mathrm{C}$ under $12 / 12$-h light/dark cycles and all tests were performed between 1000 and 1600 . The experiments were approved by the University of Haifa Ethics and Animal Care Committee, and adequate measures were taken to minimize pain or discomfort, in accordance with the guidelines laid down by the US NIH regarding the care and use of animals for experimental procedures. All the rats underwent a handling-familiarization session before the beginning of the experiment.

\section{Surgery}

The rats were anesthetized with Equithesin $\left(2.12 \% \mathrm{w} / \mathrm{v} \mathrm{MgSO}_{4}\right.$, $10 \% \mathrm{v} / \mathrm{v}$ ethanol, 39.1\% (v/v) propylene glycol, $0.98 \%(\mathrm{w} / \mathrm{v})$ sodium pentobarbital, and $4.2 \%$ (w/v) chloral hydrate) $\mathrm{L} / \mathrm{kg}$. They were restrained in a stereotaxic apparatus (David Kopf Instruments, Tujunga, CA), and implanted bilaterally with stainless steel guide cannulae (23 gauge, thin wall) aimed at the IC (anteroposterior, $+1.2 \mathrm{~mm}$ relative to bregma; lateral, $\pm 5.5 \mathrm{~mm}$; ventral, $-5.5 \mathrm{~mm}$ ) according to Paxinos and Watson (1998). The cannulae were held in place with acrylic dental cement and secured with two skull screws. A stylus was placed in the guide cannula to prevent clogging. After the surgery, the rats received antibiotic (Pen-strep 20/ 25 veterinary, $0.2 \mathrm{ml}$ ) and painkiller (Calmagine at $0.02 \mathrm{ml}$ per $100 \mathrm{~g}$ ). The animals were allowed 1 week to recuperate before being subjected to experimental manipulations.

\section{Conditioned Taste Aversion}

CTA acquisition and extinction. CTA was conducted as previously described (Akirav et al, 2006, 2009; Berman and Dudai, 2001; Eisenberg et al, 2003; Maroun et al, 2012; Rosenblum et al, 1993). Rats were water deprived for $24 \mathrm{~h}$ /day and then, over 3 consecutive days trained to drink water from two pipettes each containing $10 \mathrm{ml}$ of tap water during daily 20-min access sessions.

On the conditioning day, water was replaced with saccharin $(0.1 \% \mathrm{w} / \mathrm{v})$ during the 20 -min access session. Forty minutes later, rats received intraperitoneal injections of lithium chloride ( $\mathrm{LiCl}, 0.15 \mathrm{M}, 2 \%$ body weight) to induce malaise. During 2 days following training animals received a daily portion of water in two pipettes, each containing $10 \mathrm{ml}$ (Akirav et al, 2006; Bahar et al, 2003; Berman and Dudai, 2001).

On the third day, rats were given a retrieval session (T1) entailing 10 -min access to two pipettes of saccharin (each containing $10 \mathrm{ml}$ ) followed by 10 -min access to two pipettes of water (each containing $10 \mathrm{ml}$ ). This test served to examine the success of the CTA memory formation. To examine extinction of CTA, two additional and similar sessions were conducted over the next 2 days (T2, T3; detailed protocol in Maroun et al, 2012).

Weak CTA. The protocol of weak CTA was similar to regular CTA; however, animals were injected with a lower dose of $\mathrm{LiCl}$ (0.075 M, 2\% body weight; Ballarini et al, 2009).

Strong CTA. The saccharin- $\mathrm{LiCl}$ association was repeated twice on 2 consecutive days (Ivanova and Bureš, 1990; Berman and Dudai, 2001).

Reinstatement of CTA. Twenty-four hours following the last testing session (T3), animals were re-injected with $\mathrm{LiCl}$ to reinstate aversion and three hours later they had the daily ration of water (Schachtman et al, 1985; Shema et al, 2007). On the next day, the aversion index to saccharin was re-measured as above.

CTA index. CTA was measured as an aversion index, defined as $\mathrm{ml}$ of water consumption/total fluid consumption (water + saccharin $) \times 100$, with a score of 100 indicating complete CTA and a score of 50 indicating no CTA and no preference for saccharin.

\section{Microinjection}

Microinjection into the IC was preformed through a 28gauge injection needle placed in a guide cannula after removal of the dummy. The injection needle was connected via PE20 tubing to a Hamilton micro-syringe driven by a microinfusion pump (Harvard PHD 2000). In all, $1 \mu \mathrm{l}$ was injected into each hemisphere over $1 \mathrm{~min}$. The injection needle remained in the guide cannula for an additional minute to avoid dragging the injected fluid out.

\section{Pharmacology}

The PI3K inhibitor, LY294002 (2-(4-morphlinyl)-8-phenyl$4 \mathrm{H}$-1-benzopyran-4-one), a specific and cell-permeable inhibitor of PI3K (Sigma-Aldrich, Israel; Barros et al, 2001; Horwood et al, 2006; Vlahos et al, 1994) (10 mM solution in DMSO) was dissolved in saline and brought to the final concentration of $25 \mu \mathrm{M}$. Control rats were injected with vehicle (saline with DMSO).

\section{Biochemistry}

Collection of tissue samples. Twenty minutes following completion CTA acquisition (CTA), CTA retrieval (T1) or extinction (T2), animals were decapitated and the brains were frozen in liquid nitrogen and stored at $-80{ }^{\circ} \mathrm{C}$. This time point was chosen according to a previous work of Yefet et al (2006) in which the authors analyzed the time course of AKT phosphorylation following novel-taste learning, and found that in the hippocampus there is a clear peak of AKT phosphorylation $20 \mathrm{~min}$ after novel-taste learning. The brains were then sliced on a cryostat (Leica CM1900) and samples of the IC were collected using a tissue curer $1 \mathrm{~mm}$ in diameter (FST Germany) from slices obtained at anteroposterior 1.2 from bregma. 
The obtained samples were homogenized in a lysis buffer (10 mM HEPES pH 7.6, 2 mM EDTA. 2 mM EGTA pH 8.0, $0.5 \mathrm{mM}$ DTT, $1 \mathrm{mg} / \mathrm{ml}$ leupeptin, $1.6 \mathrm{mg} / \mathrm{ml}$ aprotonin, $5 \mathrm{mM}$ $\mathrm{NaF}, 0.1 \mathrm{mM}$ sodium orthovanadate, $10 \mathrm{mM}$ sodium pyrophosphate) using a glass teflon homogenizer. Protein content was determined using the Bradford reagent. The samples were diluted in SDS sample buffer $(62.5 \mathrm{mM}$ Tris$\mathrm{HCl} \mathrm{pH} \mathrm{6.8,} \mathrm{10 \%} \mathrm{glycerol,} 2.3 \%$ SDS and 5\% $\beta$-mercaptoethanol), boiled for $5 \mathrm{~min}$ and stored at $-20{ }^{\circ} \mathrm{C}$.

\section{Western Blotting}

Equal total amount of protein was loaded in each lane $(10 \mu \mathrm{g})$ of $7.5 \%$ SDS-PAGE gels. After standard electrophoresis, the proteins were transferred onto a nitrocellulose membrane $(0.45 \mu \mathrm{m}$; Whatman) and the bands were visualized using Ponceau staining (Bio-Rad). Following washing with distilled water, membranes were blocked for $1 \mathrm{~h}$ at room temperature with blocking buffer (5\% blotting milk in TBS containing $0.1 \%$ Tween-20 (TBS-T)). Membranes were reacted with the primary antibody-AKT or p-AKT (Ser473, Cell Signaling Technology) overnight at $4{ }^{\circ} \mathrm{C}$. Following washes in TBS-T, membranes were incubated with corresponding secondary antibody anti-rabbit (Jackson ImmunoResearch). Proteins were visualized by enhanced chemiluminescence (ECL Western blotting analysis system; GE Healthcare) and quantified with a chargecoupled device camera (XRS; Bio-Rad). Each sample was measured relatively to the background, and phosphorylation levels were estimated from the ratio of phosphorylated protein and total amount of the protein.

\section{Statistical Analysis}

Results are presented as aversion index and SEM. Student's $t$-test was used when appropriate. ANOVA for repeated measures test was used and followed by post hoc comparisons that were applied to all results by using the least significant difference (LSD) multiple comparison tests.

\section{RESULTS}

\section{CTA Acquisition and Extinction Are Associated with an Increase/Decrease in AKT Phosphorylation in the IC}

Animals underwent CTA and extinction according to the behavioral protocols previously described (Maroun et al, 2012). Animals were decapitated $20 \mathrm{~min}$ following CTA (CTA: $n=7$ ), the first extinction training (T1: $n=7$ ) or following two consecutive extinction training sessions (T2: $n=9)$. The matched control groups were similar to the experimental group except that they received an injection of saline instead of LiCl. As there was no significant difference between the control groups that were taken at the different testing day, they were grouped together $(n=19)$.

Samples of IC obtained after the different behavioral manipulations were subjected to western blot analysis and the results were normalized to those of saline-control rats. ANOVA showed significant differences between the groups $(F(3,38)=9.4 ; P<0.001$; Figure 1b). Post hoc analysis showed that the Saline group was significantly different from the CTA and T2 groups $(P<0.05)$ but did not differ from the $\mathrm{T} 1$ group.

The CTA group showed an increase in the phosphorylation of AKT compared with the Saline group (Saline: $1.00 \pm 0.04 ; \mathrm{LiCl}: 1.46 \pm 0.15 ; P<0.05)$. In contrast, extinction training was associated with a gradual reduction in AKT phosphorylation. Specifically, there was no difference between the two groups after the first extinction training (T1: $1.06 \pm 0.09$; Saline: $1.00 \pm 0.04 ; P>0.05$ ). However, following the second extinction training, there was a significant difference between the group that underwent two extinction sessions (T2) and the Saline and CTA groups, as the T2 group showed reduced levels of phosphorylation (T2: $0.78 \pm 0.04 ; P<0.05$ for significant difference from the Saline and CTA groups). Total amount of AKT protein (calculated as total AKT divided by actin) was not affected by any of the behavioral procedures. The results were normalized to those of control rats (CTA: $1.1 \pm 0.11, n=7$; T1: $0.99 \pm 0.06, n=7$; T2: $1.15 \pm 0.09, n=9$; Figure 1c).

To better understand whether the increase in p-AKT following CTA is due (1) to the association between saccharin and $\mathrm{LiCl},(2)$ to the mere injection of $\mathrm{LiCl}$, or (3) to the consumption of a new taste; animals were exposed to saccharin or water without the injection of $\mathrm{LiCl}$. Independent $t$-test showed no significant differences in AKT phosphorylation between the two groups that consumed saccharin or water $(t(8)=0.23$; ns), suggesting that changes in AKT phosphorylation could not be attributed to the consumption of the new taste, saccharin.

To address whether the observed effects are due to the injections of $\mathrm{LiCl}$, two groups were injected with either $\mathrm{LiCl}$ or saline, without exposure to saccharin. Independent $t$-test on the levels of AKT phosphorylation showed significant difference between the two groups injected with $\mathrm{LiCl} /$ saline $(t(6)=2.3 ; \quad P<0.05$, Saline: $1 \pm 0.09 ; \quad$ LiCl: $1.50 \pm 0.13$; Figure 1d), suggesting that the increase in AKT phosphorylation may be attributed to the injection of LiCl. Total amount of AKT protein was not affected by any of the behavioral procedures.

\section{The Effects of PI3K Inhibition in the IC on the Acquisition of CTA and Its Extinction}

The results of the previous experiment indicate that the phosphorylation of AKT is increased following CTA and decreased following extinction; this suggests that inhibition of AKT phosphorylation is hypothesized to affect CTA acquisition and extinction. To test this, PI3K inhibitor LY294002 was microinfused into the IC at different time points to examine its effects on CTA and extinction.

However, first we sought to confirm that the microinfusion of LY294002 into the IC was associated with inhibition of the phosphorylation of AKT. To that end, we used the protocol of Chen et al (2005) and hence animals were stereotaxically microinjected with either LY294002 or vehicle into the IC $20 \mathrm{~min}$ before retrieval. Samples from the IC were collected and analyzed by western blot analysis. The infusion of LY294002 reduced phosphorylation levels of AKT in the IC compared with vehicle (Saline: $1.00 \pm 0.12$; LY294002: $0.59 \pm 0.04, P<0.01)$. These results suggest that microinjection of LY294002 inhibits the phosphorylation of AKT as was previously reported (Chen et al, 2005). 

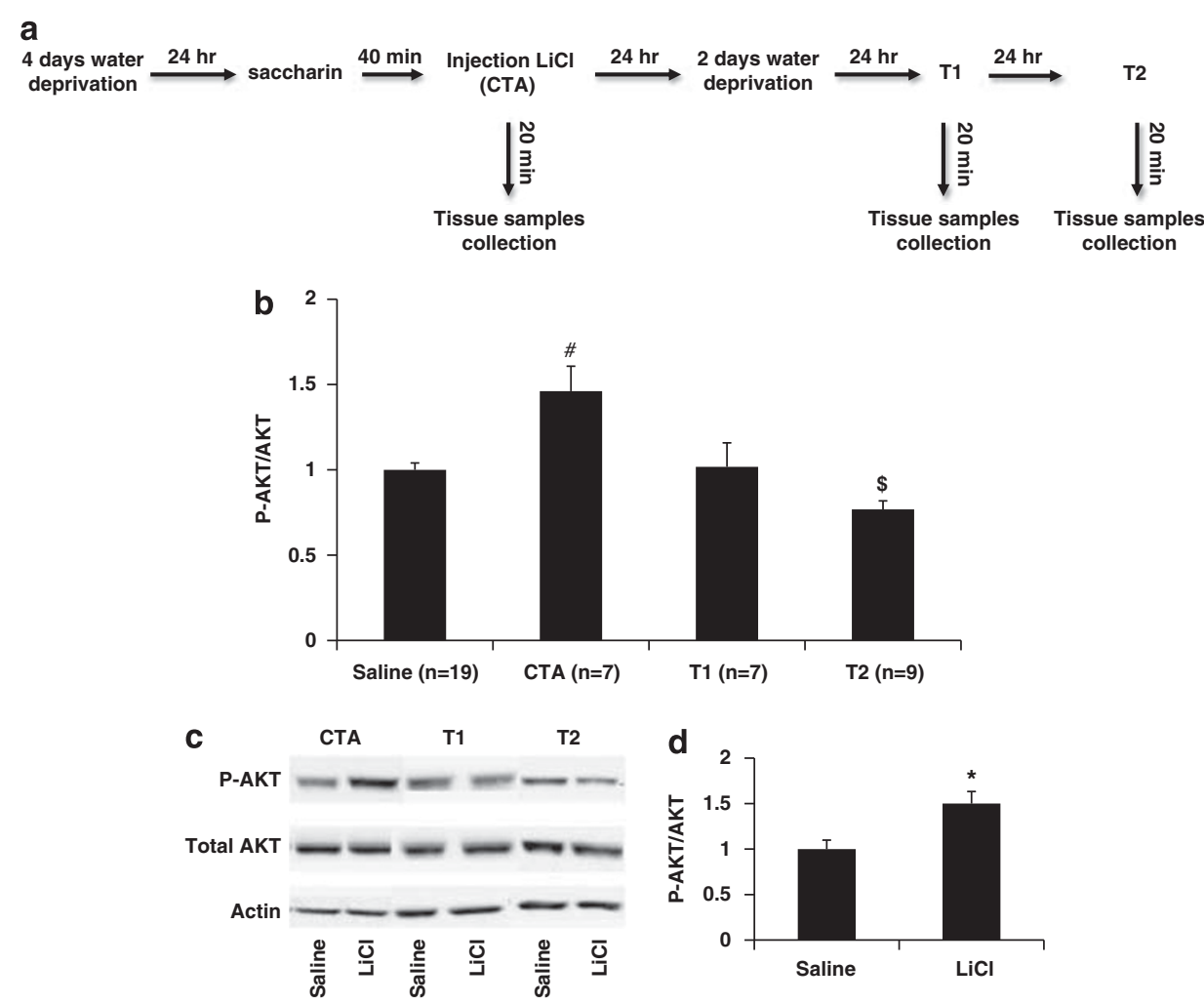

Figure I CTA acquisition and extinction are associated with an increase/decrease in AKT phosphorylation in the IC respectively. (a) Schematic representation of the experimental protocol. (b) The results point to an increase in the phosphorylation of AKT in the CTA group compared with the Saline group $(P<0.05)$. In contrast, extinction training was associated with a gradual reduction in AKT phosphorylation that was significant on T2 as the LiCl group showed reduced levels of AKT phosphorylation compared with the Saline group. (c) Representative blots of total AKT, p-AKT, and actin (after CTA (CTA); after the first and second non-reinforced tests (TI and T2, respectively)). (d) The injection of LiCl without exposure to saccharin resulted in a significant increase in the phosphorylation of AKT. \# CTA group is significantly different from the other groups $(P<0.00 \mathrm{I}) .{ }^{\$} \mathrm{~T} 2$ group is also significantly different from the saline group $(P<0.05)$. Results are represented as mean \pm SEM. *Indicates significant difference $(P<0.05)$.

\section{Inhibition of PI3K in the IC Does Not Affect the Acquisition of CTA}

To affect the acquisition of CTA, animals were microinjected with LY294002 either before saccharin consumption or after $\mathrm{LiCl}$ injection. However, as there was no significant difference between the groups, they were grouped together. Animals were microinjected with either LY294002 $(n=12)$ or vehicle $(n=9)$. The two groups did not differ in saccharin consumption (vehicle: $12.3 \pm 0.2 \mathrm{ml}$; LY294002: $\quad 11.9 \pm 0.17 \mathrm{ml}$; NS). Furthermore, ANOVA with repeated measures on the testing days did not show any significant difference between the two groups $(F(1,19)=0.09$; NS; Figure 2b). However, there was a significant effect of the testing day $(F(2,38)=121.05$; $P<0.01)$ but without a significant interaction $(\mathrm{F}(2,38)=$ $0.23, \mathrm{NS}$ ); showing that both groups extinguished similarly over the testing days.

These results suggest that PI3K in the IC is not obligatory for acquisition of CTA, as its inhibition was not associated with changes in the ability to acquire CTA.

\section{Inhibition of PI3K Reduces Aversion Without Affecting Retrieval of CTA Memory}

It was previously shown that microinjection of the PI3K inhibitor into the hippocampus or the BLA is associated with impairment in retrieval of fear memory (Chen et al,
2005; Kritman and Maroun, 2012). In the next experiment, we examined whether microinjection of LY294002 into the IC is also associated with alterations in the retrieval of CTA memory. Accordingly, LY294002 was microinjected before the first non-reinforced retrieval test (T1), which examines the success of the CTA memory formation.

The groups did not differ in saccharin consumption before LiCl injection (Vehicle $(n=9): 10.6 \pm 0.15 \mathrm{ml}$; LY294002 $(n=9): 11.1 \pm 0.17 \mathrm{ml}, t$-test: $P>0.05)$ or in total fluid consumption during the testing days $(F<1)$. However, microinjection of LY294002 before T1 was associated with significant differences between the groups $(F(1,16)=7.01$, $P<0.01$; Figure $3 \mathrm{~b}$ ). There was also a significant effect of the extinction session $(F(2,32)=112.2, P<0.01)$ and significant interaction $(F(2,32)=112.2, \quad P<0.05)$. Analysis of the results using independent $t$-test showed that administration of LY294002 into the IC was associated with reduced aversion on the T2 and T3 training sessions $(P<0.05)$, but not $\mathrm{T} 1$, as compared with the vehicle group, demonstrating that the inhibition of the PI3K does not affect the retrieval of CTA memory.

To rule out that the lack of effect of LY294002 on retrieval on T1 is due to a ceiling effect of the aversion index, we induced a weak CTA (see Materials and methods) and animals were microinfused with LY294002 before T1. Independent $t$-test on the aversion index on $\mathrm{T} 1 \mathrm{did}$ not show significant differences between the two groups (Vehicle $(n=6): 83.3 \pm 4.2 \%$; LY294002 $(n=6): 81.8 \pm 4.8 \%, P>0.05)$, 
a

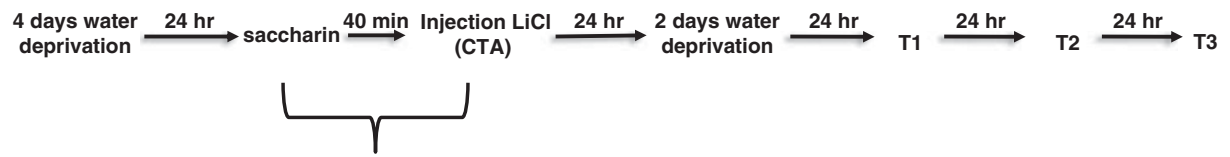

Infusion of PI3K inhibitor

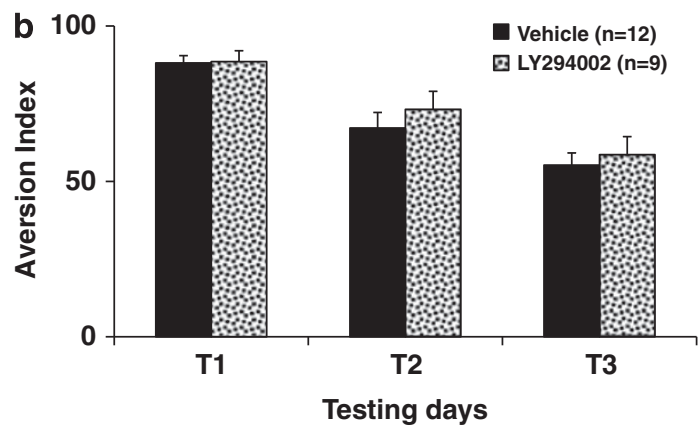

Figure 2 Inhibition of PI3K in the IC does not affect the acquisition of CTA. (a) Schematic representation of the experimental protocol. (b) Animals were stereotaxically microinjected with either LY294002 (LY294002, $n=12$ ) or vehicle (Vehicle, $n=9$ ) following the consumption of saccharin. No differences between the two groups were observed $(F(I, 19)=0.09$, NS). Furthermore, CTA memory was extinguished similarly across the extinction days as no interaction was observed $(F(2,38)=0.23, N S)$.
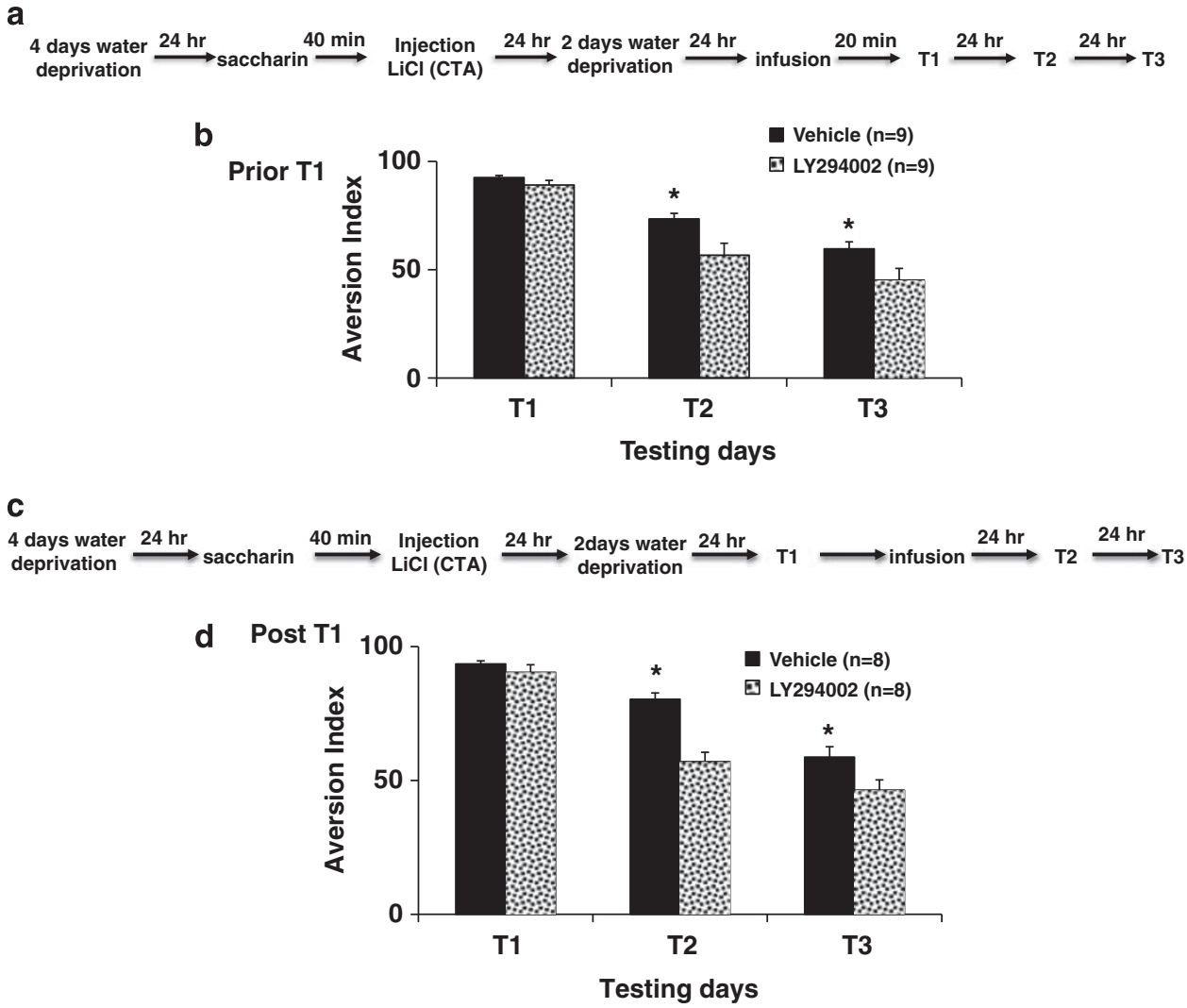

Figure 3 Inhibition of PI3K results in long-term reduction of the aversion index without affecting retrieval of CTA memory. (a, c) Schematic representation of the experimental protocol. (b) The PI3K inhibitor was microinfused into the IC before the first non-reinforced retrieval test. There are significant differences between the groups $(P<0.01)$. The groups differed on T2 $(P<0.05)$ and T3 $(P<0.05)$ but not on TI (NS). The aversion index was reduced in the group that received the LY294002 into the IC as compared with the vehicle-treated group only on T2 and T3. (d) Animals were injected with the drugs following the first retrieval session. The aversion index was less in LY294002-treated animals than in vehicle-injected rats, and both groups showed significant reductions in the index across training blocks. Results are represented as mean \pm SEM. *Indicates significant difference $(P<0.05)$.

suggesting that the absence of effect on the retrieval of CTA memory cannot be attributed to a ceiling effect.

The results of the previous experiment show that LY294002 did not affect the retrieval phase but resulted in reduction in aversion index on subsequent days. The microinjection of LY294002 before the first non-reinforced retrieval test (T1) could target two processes: the retrieval and the reconsolidation of the original CTA memory (Dudai, 2006; Miller and 
Matzel, 2000; Morris et al, 2006; Nader et al, 2000). As there was no effect on retrieval, then it is plausible that there was an effect on reconsolidation. To examine the effect of PI3K on the reconsolidation phase, animals were microinjected with the PI3K inhibitor immediately after the first retrieval session (Berman and Dudai, 2001; Eisenberg et al, 2003; Nader, 2003; Shema et al, 2007; Figure 3c).

The groups did not differ in saccharin consumption either before LiCl injection (Vehicle $(n=8): 13.0 \pm 0.94 \mathrm{ml}$; LY294002 $(n=8): 12.7 \pm 0.59 \mathrm{ml}$, $t$-test: $P>0.05)$ or during the retrieval test before the injection of drugs ( $t$-test: $P>0.05$; Figure $3 \mathrm{~d}$ ). Effects of drug and testing days (T2 and T3) on the aversion index were analyzed using a two-factor ANOVA, with repeated measures for testing session.

There were significant effects of group $(F(1,14)=19.07$, $P<0.01)$, testing session $(F(1,14)=36.6, P<0.01)$, but not significant interaction $(F<1)$. The aversion index was lower in LY294002-treated animals than in vehicle-injected ones, and both groups showed significant reductions in aversion index across training blocks (Figure 3d). This demonstrates that LY294002 microinjection into the IC post-retrieval of CTA memory on $\mathrm{T} 1$ resulted in reduced aversion during subsequent testing sessions.

\section{PI3K Inhibition After the Retrieval of Extinction Memory Has No Effect on Subsequent Extinction Memory}

The results of the previous experiment show that PI3K inhibition either before or immediately after $\mathrm{T} 1$ results in reduced aversion in the subsequent testing days (T2 and T3). Two possible explanations for this finding are that the microinjection of LY294002 post-retrieval (1) disrupted reconsolidation of the original CTA memory (for example, Nader et al, 2000; Sara, 2000; Dudai, 2004) or (2) facilitated extinction of CTA (Baumgärtel et al, 2008). To differentiate between these effects, animals were microinjected into the IC immediately after the second extinction session T2 with either vehicle $(n=10)$ or LY294002 (LY294002 post-T2, $n=9$; (Figure 4a). The underlying hypothesis was that if PI3K inhibition facilitates extinction, then microinfusion of LY294002 post-T2, when extinction is starting to be established and the aversion index is reduced will accelerate extinction.

No significant difference was found between the groups on $\mathrm{T} 1$ or $\mathrm{T} 2$ before the injections $(F(1,17)<1$, NS), however, there was a significant effect of testing days, suggesting that the CTA memory was extinguished across the days in both groups $(F(1,17)=44.4, \quad P<0.05)$. Namely, on T2 the aversion index of both groups was significantly different from T1 (Vehicle: 78.3 \pm 3.9; LY294002: 74.1 \pm 3.7 ). There was no significant interaction between groups and testing days, suggesting that the CTA memory was similarly extinguished in both groups across the two testing days (Figure 4b).

Independent $t$-test on the aversion index on T3 showed no significant differences between the two groups and both groups showed similar aversion index values $(t(17)=1.3$, NS). These results suggest that the inhibition of PI3K does not accelerate extinction.

It could be argued that the lack of effect could be due to floor effects associated with already reduced aversion index due to extinction. To rule out this, we added additional groups in which we induced strong CTA, and animals were microinjected with either vehicle $(n=8)$ or LY294002 $(n=7)$ post-T2. ANOVA with repeated measures analysis on the testing days did not show significant differences between groups $(F(1,13)=0.28$, NS). Furthermore, there was no significant effect of testing days $(F(1,13)=3.5 ; P=0.082)$, as both groups showed high aversion index over the days (T3: Vehicle: $97.2 \pm 1.5 \%$; LY294002: $98.6 \pm 1.6 \%$, NS).

Together, this set of experiments suggests that LY294002 microinjected into the IC after the second non-reinforced retrieval session does not affect subsequent extinction.

\section{PI3K Inhibition Before or After the First Non-Reinforced Retrieval Test Is Associated with Erasure of Aversion}

The results of the previous experiments show that (1) microinjection of the PI3K inhibitor after the second nonreinforced retrieval session has no effect on subsequent extinction, suggesting that the PI3K is not involved at this stage of learning, (2) the inhibition of PI3K before the first

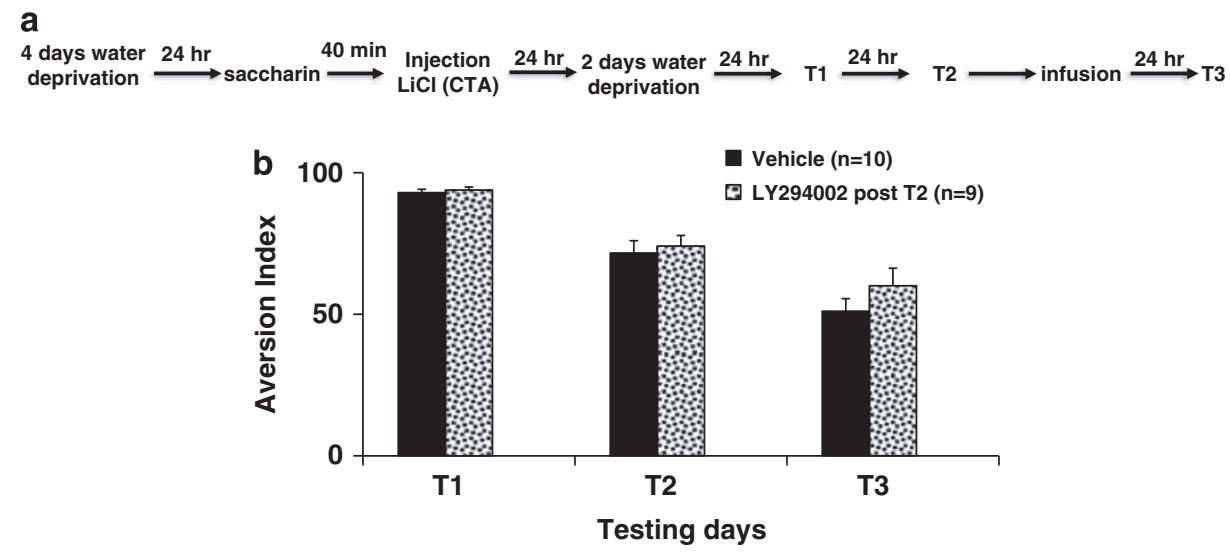

Figure 4 PI3K inhibition after the retrieval of extinction memory has no effect on the consolidation of extinction memory. (a) Schematic representation of the experimental protocol. (b) Animals were microinjected with either LY294002 (LY294002 post-T2, $n=9$ ) or vehicle (Vehicle, $n=10)$ into the IC after the retrieval of extinction memory on T2. There was no significant difference between the groups in any of the tests. Results are represented as mean \pm SEM. 
non-reinforced retrieval of CTA memory has no effect on retrieval, but on subsequent extinction sessions, and (3) inhibition of PI3K following the first non-reinforced retrieval session is associated with reduction in aversion. Based on these observations, it could be suggested that the injection of PI3K inhibitor into the IC before or immediately after the retrieval of CTA memory which resulted in decrease in the aversion index on subsequent testing sessions erases the original memory trace of CTA following its re-activation.

After extinction of the conditioned stimulus, re-exposure to the US alone can partially restore the response to the conditioned response (Rescola and Heth, 1975), suggesting that the memory of extinction is not permanent, and that the memory of the original trace is saved and not forgotten (Schachtman et al, 1985). To examine whether PI3K inhibition results in erasure of the original CTA memory, animals were microinjected with LY294002/vehicle either before $\mathrm{T} 1$ or immediately after $\mathrm{T} 1$ as described in the previous experiment (Figure 3), and following extinction training they received an additional reminder in the form of an injection of LiCl (Schachtman et al, 1985; Shema et al, 2007) The aversion to saccharin was tested $24 \mathrm{~h}$ later (Figure 5a for experimental timeline).

Consistent with the previous experiment, there was no significant difference between the groups on $\mathrm{T} 1$ $(\mathrm{F}(2,34)=1.03, \mathrm{NS}$; Figure $5 \mathrm{~b})$ suggesting that the injection of LY294002 before T1 did not affect the retrieval of CTA.

In contrast, ANOVA with repeated measures on $\mathrm{T} 2$ and T3 showed significant differences between the groups $(F(2,34)=5.94 ; P<0.01$; Figure $5 \mathrm{~b})$, significant effect of testing day $(\mathrm{F}(2,34)=127.0 ; P<0.01)$, but without significant interaction of the group and testing days $(F<1)$.
Post hoc analysis showed that LY294002 groups were significantly different from the vehicle-treated group, as they showed reduced aversion index.

ANOVA on the aversion index $24 \mathrm{~h}$ after the application of the reminder ( $\mathrm{T} 4$ post-reinstatement; injection of $\mathrm{LiCl}$ ) showed significant differences between the groups $(F(2,34)=5.9 ; P<0.01)$ and post hoc analysis showed that the groups that were injected with LY294002 were significantly different from the vehicle group. Specifically, in contrast to the Vehicle group that did not show further decrease in the aversion after the application of the reminder and even showed a slight recovery of the extinguished CS, the LY294002-treated group expressed low aversion index levels (Vehicle: $58.29 \pm 6.18 \%$; LY294002 prior T1: $36.7 \pm 7.6 \%$; LY294002 post-T1: $33.5 \pm 3.62 ; P<0.05$ for significant difference from the vehicle group; Figure $5 b$ ).

There was no significant difference in total fluid consumption across the testing days $(F<1)$, suggesting that the differences in the aversion index could not be attributed to increased preference. Together, the results suggest differential kinetics of extinction in the different groups, and show that the inhibition of PI3K prevented the recovery of the original CTA memory.

\section{DISCUSSION}

The major finding of this study is that inhibition of PI3K phosphorylation in the IC before or after the first nonreinforced retrieval test is associated with long-term erasure of the original CTA memory trace. Interestingly, microinjection of the PI3K inhibitor is not associated with impairments in the retrieval of the conditioned taste

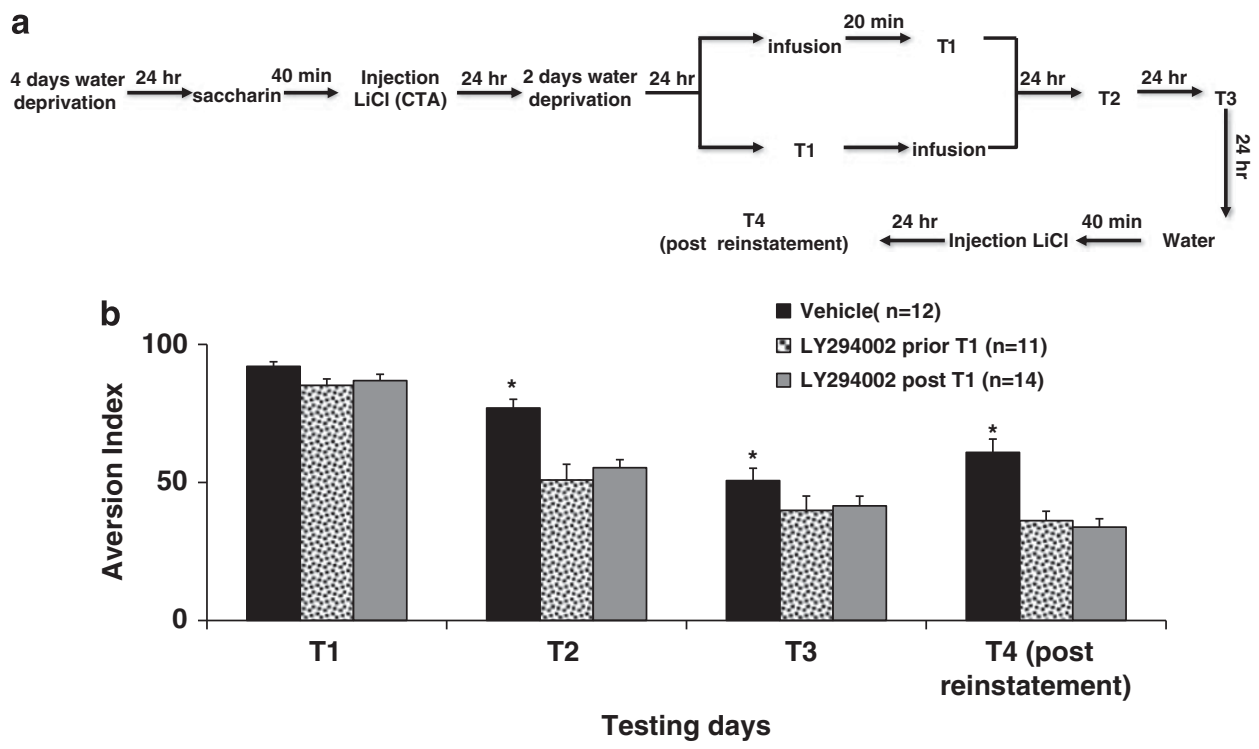

Figure $5 \mathrm{PI} 3 \mathrm{~K}$ inhibition before or after the first non-reinforced retrieval test is associated with erasure of the original CTA memory. (a) Schematic representation of the experimental protocol. (b) Animals were microinjected either before TI or immediately after TI with PI3K inhibitor or vehicle and following extinction training they received an additional reminder as an injection of $\mathrm{LiCl}$ and $24 \mathrm{~h}$ later the aversion index for saccharin was tested. There was no significant difference between the groups on TI. In contrast, there was a significant difference between the groups on T2 and T3, the LY-treated groups showing reduced aversion index to the saccharin compared with the vehicle-treated animals. Twenty-four hours following the reinstatement, the LY-treated groups expressed low and similar aversion index values in comparison with the Vehicle group which showed recovery of CTA. * Indicating significant differences $(P<0.05)$. Results are represented as mean \pm SEM. 
response, but rather affects the consolidation phase, as the effect is evident $24 \mathrm{~h}$ after injection. The effect on consolidation was confirmed by the inhibition of PI3K immediately after the first non-reinforced retrieval test (for example, Berman and Dudai, 2001; Eisenberg et al, 2003; Milekic and Alberini, 2002; Nader et al, 2000).

Another important finding of this study is related to the bidirectional and differential expression of phosphorylated AKT following CTA acquisition and extinction, which is consistent with previously reported data (Chen et al, 2005; Lin et al, 2001, 2003). Surprisingly, the microinjection of the PI3K inhibitor LY9420042 did not prevent CTA acquisition or extinction, suggesting that PI3K cascade is not obligatory for these processes.

\section{Inhibition of PI3K and Possible Role in Reconsolidation}

The results show that the microinjection of the PI3K inhibitor before the first non-reinforced retrieval of the CTA memory did not affect retrieval of the CTA memory but rather, the effect was observed $24 \mathrm{~h}$ later suggesting an effect on reconsolidation rather than on retrieval. The specific effect of PI3K inhibition on the reconsolidation phase was confirmed by microinjection of LY9420042 immediately following the first non-reinforced retrieval of the CTA memory trace (eg, Akirav et al, 2006; Berman and Dudai, 2001; Eisenberg et al, 2003), which resulted in decrease in the aversion index. Accordingly, these observations suggest that PI3K inhibition either affected reconsolidation or alternatively, its inhibition resulted in facilitated extinction of CTA. These predictions are based on many previous studies showing that memory retrieval may initiate two potentially dissociable but opposite processes: reconsolidation and extinction. It has been suggested that reconsolidation acts on the original memory (Milekic and Alberini, 2002; Nader et al, 2000; Taubenfeld et al, 2001), whereas extinction involves the formation of distinct alternative (CS-no US) memories (Quirk et al, 2000). Reconsolidation acts to stabilize, whereas extinction tends to weaken the expression of the original memory (Suzuki et al, 2004). Furthermore, using the PI3K inhibitor, intervention at this time point (after the first non-reinforced retrieval test) was shown to affect the reconsolidation of the original memory trace (Alberini, 2005; Berman and Dudai, 2001; Eisenberg et al, 2003; Milekic and Alberini, 2002; Nader et al, 2000).

Thus, in order to differentiate between whether the observed effect of LY294002 (applied either before or after the first non-retrieval test), which resulted in attenuation of the aversion index is due to facilitation of extinction or due to the disruption of reconsolidation leading to erasure of the original memory, animals were re-injected with $\mathrm{LiCl}$ to reinstate aversion (for example, Shema et al, 2007). We hypothesized that if the attenuation of aversion was due to facilitation of extinction, then reinstatement of CTA will indeed result in recovery of the conditioned response, as extinction does not erase the original memory (Bouton, 1993; Konorski, 1948; Pavlov, 1927; Wagner, 1981). Alternatively, if the original conditioned response did not recover following reinstatement, then this would indicate that LY294002 erased the original CTA memory. Indeed, the results confirmed the latter hypothesis as the LY294002- treated animals did not show return of the aversion index, unlike the vehicle-treated animals that had an increase in the aversion index.

It could be claimed that there was not a full recovery of the extinguished CS response in the vehicle-treated animals as the aversion index was not fully recovered. These results are in line with (1) the idea that following extinction of the conditioned stimulus, re-exposure to the US alone can partially restore the response to the conditioned response (Rescola and Heth, 1975) and (2) previously reported data that do not show full recovery of the CS (Shema et al, 2007). Specifically in the later study by Shema et al (2007), reinstatement of CTA induced a recovery of the aversion index up to $60 \%$. These differences in reinstatement between fear and aversive-based paradigms could be due to the fact that each of these paradigms depends on different molecular, cellular, and neural bases (for review, Myers and Davis, 2002).

Together, our results provide additional evidence to support the role of this cascade in the maintenance of aversive memories (Kritman and Maroun, 2012). In contrast, inhibition of PI3K phosphorylation following the second test at which the extinction is already established did not result in subsequent facilitation of extinction consolidation; this was confirmed by inducing a strong CTA protocol to exclude floor effect. These results indicate that in contrast to its effect on the reconsolidation of CTA, the PI3K is not involved in the consolidation of extinction of CTA and its inhibition does not result in altered extinction.

Several studies have previously shown that if re-exposure is very short (without accompanying extinction), reconsolidation will predominate and blockers will cause low levels of the original CS (impaired reconsolidation). If re-exposure is long enough to induce extinction, then extinction will predominate and blockers will cause maintained response to CS (impaired extinction). Thus, an extinction session may initially trigger reconsolidation, but this leads to consolidation of extinction as the session progresses (reviewed in Quirk and Mueller, 2008). It is worthy to note that in the present study the single retrieval in the absence of the US did not result in significant extinction as the aversion is strong and thus we tend to conclude that the effect could be on reconsolidation, though that we cannot fully rule out the initiation of extinction acquisition. However, when PI3K was inhibited when extinction is already initiated (after T2), there was no effect on subsequent extinction. Hence, our results give the first evidence that demonstrates that the activation of $\mathrm{AKT}$ is required for the reconsolidation of CTA but not for consolidation of extinction of CTA.

\section{PI3K and Retrieval of CTA Memory}

The lack of effect of LY294002 on retrieval is inconsistent with previous reports from other laboratories and ours (Chen et al, 2005; Kritman and Maroun, 2012). Specifically, Chen et al (2005) showed that when LY294002 was microinjected into the CA1 region in the hippocampus it transiently attenuated the retrieval of contextual fear conditioning, and the animals exhibited reduced freezing levels during the retrieval test. In our recent study in which we used the contextual fear conditioning paradigm, microinjection of LY294002 before the retrieval of fear memory 
was associated with fear memory attenuation which persisted $24 \mathrm{~h}$ after injection, suggesting that the inhibition of PI3K disrupted retrieval and reconsolidation (Kritman and Maroun, 2012). In the present experiment, the effect of LY294002 was limited to reconsolidation, as the effect was observed only $24 \mathrm{~h}$ later without an effect on the retrieval when it was microinjected before T1. We confirmed the absence of effect of PI3K inhibition on retrieval of CTA by using a weak CTA protocol to rule out that the lack of effect on retrieval is due to a ceiling effect. Indeed, PI3K inhibition did not affect retrieval of weak CTA memory; confirming that PI3K is not involved in the retrieval of CTA memory. However, consistent with the former studies (Chen et al, 2005; Kritman and Maroun, 2012), the present report shows attenuation of the aversion index following the microinfusion of LY294002.

The differences between the two paradigms of contextual fear conditioning and CTA could be due to the fact that each of these paradigms recruits its own constellation of cellular and molecular basis (for review, Davis and Myers, 2002; Myers and Davis, 2002). We have previously reported differences between these two paradigms (Akirav et al, 2009) and showed that while the extinction in both paradigms is impaired following exposure to a behavioral stressor, the reversal of stress-induced impairments is not dependent on the same mechanisms. However, further studies should explore the differences between the mechanisms mediating extinction of these types of memories.

Together, the present findings and our previously reported results (Kritman and Maroun, 2012) show that in both CTA and contextual fear conditioning the inhibition of PI3K before or after the first retrieval test is associated with reduction in the conditioned response that is observed $24 \mathrm{~h}$ after microinjection, suggesting a prolonged window for the effects of PI3K inhibition on reconsolidation.

The present findings provide additional evidence to our previous work, suggesting that targeting PI3K has differential effects depending on the temporal and spatial parameters of time and site of injection (Kritman and Maroun, 2012).

\section{Changes in AKT Following CTA Acquisition and Extinction Are Not Obligatory for the Memory Formation}

The presumed involvement of AKT in synaptic plasticity and memory processes suggests that its phosphorylation levels should change when learning occurs. To evaluate the effect of CTA on phosphorylation levels of AKT in the IC, tissues were collected following CTA acquisition and extinction. The results show differential activation of AKT following CTA conditioning and extinction. Specifically, in the IC the levels of p-AKT were dramatically increased after CTA conditioning as compared with the control animals, and extinction training was associated with dephosphorylation of AKT following the extinction session. These results are consistent with the study of Lin et al (2003), which showed similar results following fear conditioning paradigms.

The changes in AKT could not be attributed to the exposure to a novel taste, consistent with a previous report that did not show changes in AKT phosphorylation in the IC (Yefet et al, 2006).
However, we show that the changes in AKT phosphorylation following CTA could result from the mere injection of $\mathrm{LiCl}$ as was previously reported (Lamprecht and Dudai, 1995). This may explain the reason why the inhibition of the PI3K at different stages that are critical for the formation of CTA did not affect CTA acquisition. Together, our results suggest that PI3K is not involved in CTA formation.

Extinction training is associated with reduction in AKT phosphorylation, consistent with the finding of Lin et al (2003). Surprisingly, although AKT is a downstream target of PI3K which undergoes dephosphorylation following extinction, its inhibition is not obligatory for CTA acquisition or extinction. ERK/MAPK is a parallel kinase cascade that exhibits extensive cross-talk with the PI3K cascade and both pathways can activate or inhibit each other (Aksamitiene et al, 2012). ERKI/II activity was increased in the IC after CTA conditioning (Kwon and Houpt, 2012; Swank, 2000). Inhibition of MEK, an upstream kinase of ERKI/II, by pharmacological measures in the IC attenuated CTA memory (Berman et al, 1998; Kwon and Houpt, 2012). Thus, it is plausible that ERK/MAPK pathway compensates for the inhibition of the PI3K cascade in the process of CTA acquisition or extinction.

It is well established that protein synthesis and different intracellular signaling pathways are crucial for long-term memories of both fear and taste aversion conditioning (Houpt and Berlin, 1999). For example, $\mathrm{Ca}^{2+} /$ phospholipiddependent protein kinase (PKC) has been reported to be necessary in the IC for formation of a CTA memory (NunezJaramillo et al, 2007; Sacchetti and Bielavska, 1998; Yasoshima and Yamamoto, 1997). Overexpression of PKC isoform $\mathrm{M}$ zeta $(\mathrm{PKM} \zeta)$ led to enhancement of long-term CTA memory, whereas inhibition of $\mathrm{PKM} \zeta$ led to erasure or of long-term CTA memory (Edry et al, 2011; Shema et al, 2007, 2009, 2011). Further, the inhibition of $\mathrm{PKM} \zeta$ in the amygdala also resulted in attenuated CTA memory (Gámiz and Gallo, 2011).

Protein kinase A (PKA), a serine/threonine kinase has been shown to be activated by CAMP. Infusions of 8-bromoadenosine $3^{\prime}-5^{\prime}$ cyclic monophosphate (8-Br-cAMP), an analog of the second messenger cAMP into the IC just before $\mathrm{LiCl}$ administration enhanced CTA retention. Inhibition of PKA at the time of retrieval of CTA in the BLA resulted in rapid extinction (Koh et al, 2002; Miranda and McGaugh, 2004).

All in all, our present data add new evidence to suggest that PI3K is engaged in consolidation of aversive memories, as its inhibition in the IC leads to erasure of CTA memory. The study of acquisition and extinction of aversive memories is crucial for anxiety disorders, as one of the major characteristics of PTSD patients and of animal models of PTSD is extinction impairment (Frankland et al, 2006; Mamiya et al, 2009; Rosenblum et al, 1995). Current treatments for PTSD include exposure therapies that incorporate extinction procedures (Kelly and Lynch, 2000). The PI3K/ AKT/mTOR kinase cascade has been targeted in developing therapies for cancer (Russo et al, 2005) and neurological disorders (Baskin et al, 1988) such as epilepsy (Czech, 1989). Based on the present study and our previous work (Kritman and Maroun, 2012), the PI3K may serve as the target for the erasure of fear and aversive memories. 


\section{ACKNOWLEDGEMENTS}

This work was supported by Israel Science Foundation grant to MM. The authors thank Drs Guillaume Ferreira and Shunit Gal Ben-Ari for valuable comments.

\section{DISCLOSURE}

The authors declare no conflict of interest.

\section{REFERENCES}

Akirav I, Khatsrinov V, Vouimba RM, Merhav M, Ferreira G, Rosenblum $\mathrm{K}$ et al (2006). Extinction of conditioned taste aversion depends on functional protein synthesis but not on NMDA receptor activation in the ventromedial prefrontal cortex. Learn Mem 13: 254-258.

Akirav I, Segev A, Motanis H, Maroun M (2009). D-Cycloserine into the BLA reverses the impairing effects of exposure to stress on the extinction of contextual fear, but not conditioned taste aversion. Learn Mem 16: 682-686.

Aksamitiene E, Kiyatkin A, Kholodenko BN (2012). Cross-talk between mitogenic Ras/MAPK and survival PI3K/Akt pathways: a fine balance. Biochem Soc Trans 40: 139-146.

Alberini CM (2005). Mechanisms of memory stabilization: are consolidation and reconsolidation similar or distinct processes? Trends Neurosci 28: 51-56.

Bahar A, Samuel A, Hazvi S, Dudai Y (2003). The amygdalar circuit that acquires taste aversion memory differs from the circuit that extinguishes it. Eur J Neurosci 17: 1527-1530.

Ballarini F, Moncada D, Martinez MC, Alen N, Viola H (2009). Behavioral tagging is a general mechanism of long-term memory formation. Proc Natl Acad Sci USA 106: 14599-14604.

Barros DM, Mello e Souza T, de Souza MM, Choi H, DeDavid e Silva T, Lenz G et al (2001). LY294002, an inhibitor of phosphoinositide 3-kinase given into rat hippocampus impairs acquisition, consolidation and retrieval of memory for one-trial step-down inhibitory avoidance. Behav Pharmacol 12: 629-634.

Baskin DG, Wilcox BJ, Figlewicz DP, Dorsa DM (1988). Insulin and insulin-like growth factors in the CNS. Trends Neurosci 11: 107-111.

Baumgärtel K, Genoux D, Welzl H, Tweedie-Cullen RY, Koshibu K, Livingstone-Zatchej $\mathrm{M}$ et al (2008). Control of the establishment of aversive memory by calcineurin and Zif268. Nat Neurosci 11: 572-578.

Berman DE, Hazvi S, Stehberg J, Bahar A, Dudai Y (2003). Conflicting processes in the extinction of conditioned taste aversion: behavioral and molecular aspects of latency, apparent stagnation, and spontaneous recovery. Learn Mem 10: 16-25.

Berman DE, Dudai Y (2001). Memory extinction, learning anew, and learning the new: dissociations in the molecular machinery of learning in cortex. Science 291: 2417-2419.

Berman DE, Hazvi S, Neduva V, Dudai Y (2000). The role of identified neurotransmitter systems in the response of insular cortex to unfamiliar taste: activation of ERK1-2 and formation of a memory trace. J Neurosci 20: 7017-7023.

Berman DE, Hazvi S, Rosenblum K, Seger R, Dudai Y (1998). Specific and differential activation of mitogen-activated protein kinase cascades by unfamiliar taste in the insular cortex of the behaving rat. J Neurosci 18: 10037-10044.

Bouton ME (1993). Context, time, and memory retrieval in the interference paradigms of Pavlovian learning. Psychol Bull 114: 80.

Bouton ME, Nelson JB (1994). Context-specificity of target versus feature inhibition in a feature-negative discrimination. $J$ Exp Psychol Anim Behav Process 20: 51-65.
Bures J, Bermúdez-Rattoni F, Yamamoto T (1998). Conditioned Taste Aversion: Memory of a Special Kind. Oxford University Press: Oxford, UK.

Bureš J, Burešová O, Křivánek J (1988). Brain and Behavior: Paradigms for Research in Neural Mechanisms. Wiley: New York, NY.

Chen X, Garelick MG, Wang H, Lil V, Athos J, Storm DR (2005). PI3 kinase signaling is required for retrieval and extinction of contextual memory. Nat Neurosci 8: 925-931.

Czech MP (1989). Signal transmission by the insulin-like growth factors. Cell 59: 235-238.

Davis M, Myers KM (2002). The role of glutamate and gammaaminobutyric acid in fear extinction: clinical implications for exposure therapy. Biol Psychiatry 52: 998-1007.

Dudai Y (2004). The neurobiology of consolidations, or, how stable is the engram? Annu Rev Psychol 55: 51-86.

Dudai Y (2006). Reconsolidation: the advantage of being refocused. Curr Opin Neurobiol 16: 174-178.

Edry E, Lamprecht R, Wagner S, Rosenblum K (2011). Virally mediated gene manipulation in the adult CNS. Front $\mathrm{Mol}$ Neurosci 4: 57.

Eisenberg M, Kobilo T, Berman DE, Dudai Y (2003). Stability of retrieved memory: inverse correlation with trace dominance. Science 301: 1102-1104.

Fanselow MS, Gale GD (2003). The amygdala, fear, and memory. Ann NY Acad Sci 985: 125-134.

Frankland PW, Ding HK, Takahashi E, Suzuki A, Kida S, Silva AJ (2006). Stability of recent and remote contextual fear memory. Learn Mem 13: 451-457.

Gámiz F, Gallo M (2011). Intra-amygdala ZIP injections impair the memory of learned active avoidance responses and attenuate conditioned taste-aversion acquisition in rats. Learn Mem 18: 529-533.

Garcia J, Ervin FR, Koelling RA (1966). Learning with prolonged delay of reinforcement. Psychon Sci 5: 121-122.

Garcia J, Kimeldorf DJ, Koelling RA (1955). Conditioned aversion to saccharin resulting from exposure to gamma radiation. Science 122: 157-158.

Horwood JM, Dufour F, Laroche S, Davis S (2006). Signalling mechanisms mediated by the phosphoinositide 3-kinase/Akt cascade in synaptic plasticity and memory in the rat. Eur $J$ Neurosci 23: 3375-3384.

Houpt TA, Berlin R (1999). Rapid, labile, and protein synthesisindependent short-term memory in conditioned taste aversion. Learn Mem 6: 37-46.

Ivanova S, Bureš J (1990). Conditioned taste aversion is disrupted by prolonged retrograde effects of intracerebral injection of tetrodotoxin in rats. Behav Neurosci 104: 948.

Kelly A, Lynch MA (2000). Long-term potentiation in dentate gyrus of the rat is inhibited by the phosphoinositide 3-kinase inhibitor, wortmannin. Neuropharmacology 39: 643-651.

Koh MT, Thiele TE, Bernstein IL (2002). Inhibition of protein kinase A activity interferes with long-term, but not short-term, memory of conditioned taste aversions. Behav Neurosci 116: 1070-1074.

Konorski J (1948). Conditioned Reflexes and Neuron Organization. Cambridge University Press: Cambridge, UK.

Kritman M, Maroun M (2012). Inhibition of the PI3 kinase cascade in corticolimbic circuit: temporal and differential effects on contextual fear and extinction. Int J Neuropsychopharmacol 1: 1-9.

Kwon B, Houpt TA (2012). Mitogen-activated protein kinase in the amygdala plays a critical role in lithium chloride-induced taste aversion learning. Neurobiol Learn Mem 97: 132-139.

Lamprecht R, Dudai Y (1995). Differential modulation of brain immediate early genes by intraperitoneal LiCl. Neuroreport 7: 289-293. 
LeDoux JE, Romanski L, Xagoraris A (1989). Indelibility of subcortical emotional memories. J Cogn Neurosci 1: 238-243.

Lin CH, Yeh SH, Leu TH, Chang WC, Wang ST, Gean PW (2003). Identification of calcineurin as a key signal in the extinction of fear memory. J Neurosci 23: 1574-1579.

Lin CH, Yeh SH, Lin CH, Lu KT, Leu TH, Chang WC et al (2001). A role for the PI-3 kinase signaling pathway in fear conditioning and synaptic plasticity in the amygdala. Neuron 31: 841-851.

Mamiya N, Fukushima H, Suzuki A, Matsuyama Z, Homma S, Frankland PW et al (2009). Brain region-specific gene expression activation required for reconsolidation and extinction of contextual fear memory. J Neurosci 29: 402-413.

Maroun M, Kavushansky A, Holmes A, Wellman C, Motanis H (2012). Enhanced extinction of aversive memories by highfrequency stimulation of the rat infralimbic cortex. PLOS ONE 7: e35853.

Milekic MH, Alberini CM (2002). Temporally graded requirement for protein synthesis following memory reactivation. Neuron 36: 521-525.

Miller RR, Matzel LD (2000). Memory involves far more than 'consolidation'. Nat Rev Neurosci 1: 214-216.

Miranda MI, McGaugh JL (2004). Enhancement of inhibitory avoidance and conditioned taste aversion memory with insular cortex infusions of 8-Br-cAMP: involvement of the basolateral amygdala. Learn Mem 11: 312-317.

Morris RG, Inglis J, Ainge JA, Olverman HJ, Tulloch J, Dudai Y et al (2006). Memory reconsolidation: sensitivity of spatial memory to inhibition of protein synthesis in dorsal hippocampus during encoding and retrieval. Neuron 50: 479-489.

Myers KM, Davis M (2002). Behavioral and neural analysis of extinction. Neuron 36: 567-584.

Nachman M (1963). Learned aversion to the taste of lithium chloride and generalization to other salts. J Comp Physiol Psychol 56: 343.

Nader K (2003). Memory traces unbound. Trends Neurosci 26: $65-72$.

Nader K, Schafe GE, Le Doux JE (2000). Fear memories require protein synthesis in the amygdala for reconsolidation after retrieval. Nature 406: 722-726.

Nunez-Jaramillo L, Delint-Ramirez I, Bermudez-Rattoni F (2007). PKC blockade differentially affects aversive but not appetitive gustatory memories. Brain Res 1148: 177-182.

Opazo P, Watabe AM, Grant SG, O'Dell TJ (2003). Phosphatidylinositol 3-kinase regulates the induction of long-term potentiation through extracellular signal-related kinase-independent mechanisms. J Neurosci 23: 3679-3688.

Pavlov I (1927). Conditioned Reflexes: An Investigation of the Physiological Activity of the Cerebral Cortex (GV Anrep, Trans.). Oxford University: London.

Paxinos G, Watson C (1998). The Rat Brain in Stereotaxic Coordinates. 4th editionAcademic Press: New York.

Quirk GJ, Mueller D (2008). Neural mechanisms of extinction learning and retrieval. Neuropsychopharmacology 33: 56-72.

Quirk GJ, Russo GK, Barron JL, Lebron K (2000). The role of ventromedial prefrontal cortex in the recovery of extinguished fear. J Neurosci 20: 6225-6231.

Rescorla RA (1996). Preservation of Pavlovian associations through extinction. Quar J Exp Psychol B 49: 245-258.

Revusky SH, Bedarf EW (1967). Association of illness with prior ingestion of novel foods. Science 155: 219-220.
Roman C, Reilly S (2007). Effects of insular cortex lesions on conditioned taste aversion and latent inhibition in the rat. Eur J Neurosci 26: 2627-2632.

Roman C, Nebieridze N, Sastre A, Reilly S (2006). Effects of lesions of the bed nucleus of the stria terminalis, lateral hypothalamus, or insular cortex on conditioned taste aversion and conditioned odor aversion. Behav Neurosci 120: 1257-1267.

Rosenblum K, Meiri N, Dudai Y (1993). Taste memory: the role of protein synthesis in gustatory cortex. Behav Neural Biol 59: 49-56.

Rosenblum K, Schul R, Meiri N, Hadari YR, Zick Y, Dudai Y (1995). Modulation of protein tyrosine phosphorylation in rat insular cortex after conditioned taste aversion training. Proc Natl Acad Sci USA 92: 1157-1161.

Russo VC, Gluckman PD, Feldman EL, Werther GA (2005). The insulin-like growth factor system and its pleiotropic functions in brain. Endocr Rev 26: 916-943.

Sara SJ (2000). Retrieval and reconsolidation: toward a neurobiology of remembering. Learn Mem 7: 73-84.

Sacchetti B, Bielavska E (1998). Chelerythrine, a specific PKC inhibitor, blocks acquisition but not consolidation and retrieval of conditioned taste aversion in rat. Brain Res 799: 84-90.

Schachtman TR, Brown AM, Miller RR (1985). Reinstatementinduced recovery of a taste- $\mathrm{LiCl}$ association following extinction. Learn Behav 13: 223-227.

Shema R, Haramati S, Ron S, Hazvi S, Chen A, Sacktor TC et al (2011). Enhancement of consolidated long-term memory by overexpression of protein kinase Mzeta in the neocortex. Science 331: 1207-1210.

Shema R, Hazvi S, Sacktor TC, Dudai Y (2009). Boundary conditions for the maintenance of memory by PKMzeta in neocortex. Learn Mem 16: 122-128.

Shema R, Sacktor TC, Dudai Y (2007). Rapid erasure of long-term memory associations in the cortex by an inhibitor of PKM zeta. Science 317: 951-953.

Suzuki A, Josselyn SA, Frankland PW, Masushige S, Silva AJ, Kida S (2004). Memory reconsolidation and extinction have distinct temporal and biochemical signatures. J Neurosci 24: 4787-4795.

Swank MW (2000). Phosphorylation of MAP kinase and CREB in mouse cortex and amygdala during taste aversion learning. Neuroreport 11: 1625-1630.

Taubenfeld SM, Milekic MH, Monti B, Alberini CM (2001). The consolidation of new but not reactivated memory requires hippocampal C/EBP $\beta$. Nat Neurosci 4: 813-818.

Vlahos CJ, Matter WF, Hui KY, Brown RF (1994). A specific inhibitor of phosphatidylinositol 3-kinase, 2-(4-morpholinyl)-8phenyl-4H-1-benzopyran-4-one (LY294002). J Biol Chem 269: 5241-5248.

Wagner AR (1981). SOP: a model of automatic memory processing in animal behavior. In: Information Processing in Animals: Memory Mechanisms. Information Processing in Animals: Memory Mechanisms. Lawrence Erlbaum Associates: Hillsdale, NJ, 85: 5-44.

Yasoshima Y, Yamamoto T (1997). Rat gustatory memory requires protein kinase $\mathrm{C}$ activity in the amygdala and cortical gustatory area. Neuroreport 8: 1363-1367.

Yefet K, Merhav M, Kuulmann-Vander S, Elkobi A, Belelovsky K, Jacobson-Pick S et al (2006). Different signal transduction cascades are activated simultaneously in the rat insular cortex and hippocampus following novel taste learning. Eur J Neurosci 24: $1434-1442$. 